\title{
A new anatomic locking plate for the treatment of posterolateral tibial plateau fractures
}

\author{
Zhen Jian $^{1 \dagger}$, Rongguang Ao ${ }^{1 \dagger}$, Jianhua Zhou', Xinhua Jiang ${ }^{1}$, Dianying Zhang ${ }^{2^{*}}$ and Baoqing Yu ${ }^{1^{*}}$
}

\begin{abstract}
Background: Posterolateral tibial plateau fractures have become more common, and their treatment is of great importance to knee function. Additionally, there is no available literature detailing specialized anatomic locking plate for tibial plateau fractures. Therefore, the aim of the study was to evaluate the safety and clinical efficacy of an innovative anatomic locking plate for treatment of posterolateral tibial plateau fractures.

Methods: Between March 2014 and January 2016, 12 patients with posterolateral tibial plateau fracture underwent surgery with the anatomic locking plate for the posterolateral tibial plateau via the posterolateral approach. Relevant operational data for clinical evaluation were collected.

Results: The mean follow-up time was 26 months, and the mean age was 35 years for 12 patients. The mean interval between the time of injury and the surgery was 6.1 days. Radiological fracture union was evident in all patients at 12 weeks. During surgery, the blood loss ranged from 100 to $300 \mathrm{~mL}$, and the duration ranged from 55 to $90 \mathrm{~min}$. The Tegner-Lysholm functional score ranged from 85 to 97 at the final follow-up. Moreover, the final Rasmussen functional score ranged from 25 to 29, and Rasmussen anatomical score ranged from 13 to 18.

Conclusions: The newly designed anatomic locking plate for the posterolateral tibial plateau provided adequate fixation along the posterolateral tibial plateau. It proved to be safe and effective in a small-sample-size population (12 patients) during a 12- to 34-month follow-up.
\end{abstract}

Keywords: Posterolateral tibial plateau fractures, Anatomic locking plate, Posterolateral approach

\section{Background}

Tibial plateau fractures are commonly encountered in clinical practice, whereas posterolateral tibial plateau fractures have rarely been reported in the past. However, posterolateral tibial plateau fractures have been reported in up to $7 \%$ of all tibial plateau fractures with increased high-energy trauma and use of computed tomography (CT) [1-3].

Good reduction and firm fixation are vital for treatment of posterolateral tibial plateau fractures. Many authors recommend surgical treatment to restore joint congruity and mechanical stability and further guarantee

\footnotetext{
*Correspondence: zdy8016@163.com; doctorybq@163.com

†Zhen Jian and Rongguang Ao contributed equally to this work.

${ }^{2}$ Department of Orthopedics, Peking University People's Hospital, Beijing 100044, China

'Department of Orthopaedics, Shanghai Pudong Hospital, Shanghai Fudan Univercity Pudong Medical Center, Shanghai 201399, China
}

the best prognosis $[4,5]$. However, the operative treatment of posterolateral tibial plateau fractures remains a challenge due to the fibular head and ligamentous structures that impede adequate exposure of the posterolateral joint surface. Although many approaches have been reported $[4,6-9]$ to achieve appropriate exposure, there is no general consensus.

Recent studies [7-9] on the treatment of posterolateral tibial plateau fractures have focused on surgical approaches. There is no literature to our knowledge about a specialized anatomic locking plate for posterolateral tibial plateau fractures. Surgeons usually have to choose buttress plate systems such as L-shaped plates and T-plates used in the fixation of distal radius. However, due to the irregular bony structure of the region, the plates often require contouring during surgery to fit the posterolateral tibial plateau, which may potentially lead to longer operative duration, higher

(c) The Author(s). 2018 Open Access This article is distributed under the terms of the Creative Commons Attribution 4.0 International License (http://creativecommons.org/licenses/by/4.0/), which permits unrestricted use, distribution, and reproduction in any medium, provided you give appropriate credit to the original author(s) and the source, provide a link to the Creative Commons license, and indicate if changes were made. The Creative Commons Public Domain Dedication waiver (http://creativecommons.org/publicdomain/zero/1.0/) applies to the data made available in this article, unless otherwise stated. 
blood loss, and decreased mechanical strength of the plate. Additionally, the proximal locking screws in these plates may not achieve the "raft" effect to support the articular surface of the posterolateral tibial plateau fracture. Therefore, details of an innovative anatomic locking plate for the posterolateral tibial plateau (Fig. 1) and the clinical outcomes were reported to evaluate the safety and clinical efficacy of this innovative plate.

\section{Methods}

\section{General data}

This retrospective study was conducted after obtaining the approval of the institutional review board. Between March 2014 and January 2016, 12 patients with a posterolateral tibial plateau fracture (ages 18-45 years) underwent surgery with the anatomic locking plate for the posterolateral tibial plateau. Patients with osteofascial compartment syndrome, open fracture, multiple trauma, pathologic fractures, autoimmune diseases, blood disorder, and surgical contraindications were excluded. All patients received anteroposterior and lateral radiographs and three-dimensional computed tomography reconstruction images of the tibial plateau before surgery. Additionally, all patients were followed up either by clinic review or by telephone as required.

According to the CT reconstruction images, the main fragments were located in the posterior half of the lateral condyle and/or a fracture line impacted the posterior aspect of the lateral plateau. According to three-column classification [10], all cases were fractures of the posterolateral column. Preoperative management included distal bony traction and splint. After the soft tissue condition was stable, all the fractures were exposed and reduced via the posterolateral approach by the same surgeon $(\mathrm{Yu})$.

\section{Plate details}

The anatomic locking plate for the posterolateral tibial plateau (Fig. 2) was designed according to the structure of the posterolateral tibial plateau in a Chinese population. It was oblique " $T$ " shaped to fit the posterolateral structure of the tibial plateau with a low profile and beveled edges. The angle between the plate head and the waist was $136^{\circ}$. It had five $30^{\circ}$ universal locking holes to ensure precise screw insertion in the head, which fixed the plate with the posterolateral side of the tibial plateau. Especially three proximal row screws paralleled with the plateau could buttress powerfully as a "raft effect." Tiny holes in the top of the plate head were used in cases of a temporary fixation or comminuted fragment fixation by K-wire insertion and capsule sutures. When plate data was transefer into 3D computer-aided design (CAD) model, several patients' knee CT data were also integrated with the CAD model, implant placement was simulated in silico to check for accuracy. When we obtained the plate patent, the plate was made by a qualified custom-made plate manufacture (WASTON Medical Appliance Co, Ltd., China). All structure was made of titanium. Finally, the plates obtained were completely consistent with our design in terms of their shape and distribution and the orientation of screws.

\section{Surgical technique and rehabilitation}

All operations were performed using spinal anesthesia or general anesthesia with the patients in the prone position, and the lower limb tourniquet was inflated.

A posterolateral incision was placed over the biceps femoris muscle. Attention must be paid to identify and dissect the peroneal nerve posterior of the biceps femoris muscle. Then gently retracted laterally for the nerve

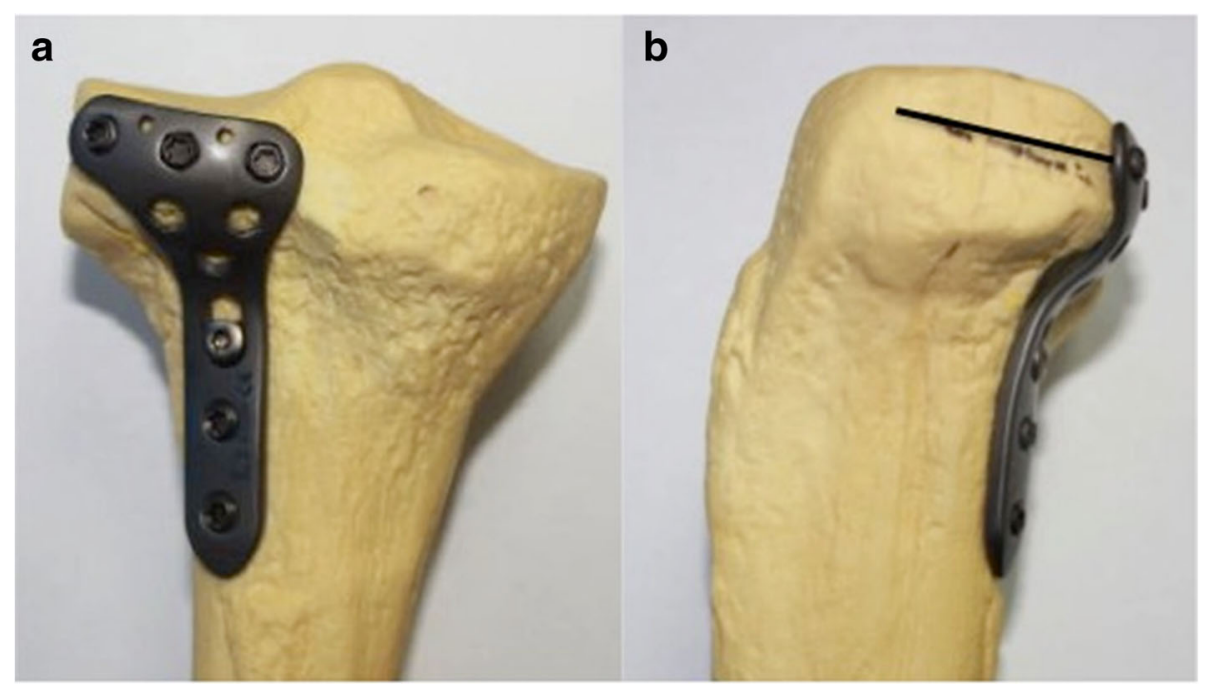

Fig. 1 The newly designed posterolateral anatomic locking plate which is specially used in posterolateral tibial plateau fracture. a, posterior view of the tibial plateau. $\mathbf{b}$, lateral view of tibial plateau 


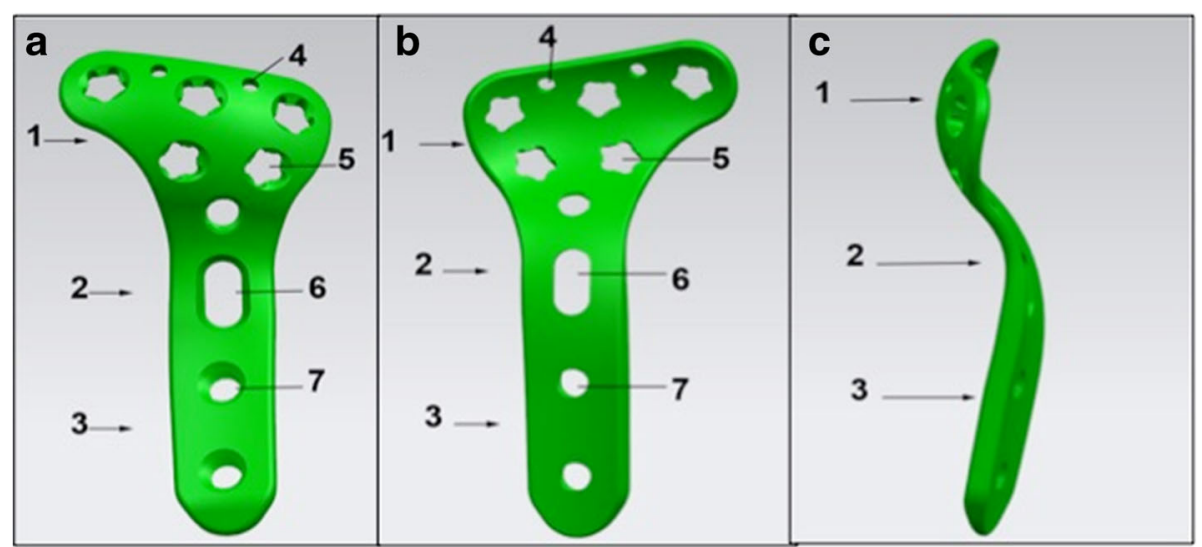

Fig. 2 Schematic figureof The newly designed posterolateral anatomic locking plate. a, posterior view; b, anterior view; c, lateral view(1.head, 2.waist, 3.body, 4.K-wires hole, 5.locking screw hole, 6.sliding compression hole, 7.locking screw hole)

protection during the operation. Medially retracted the lateral head of the gastrocnemius with the origin of the soleus elevated from the proximal section of tibia. The popliteus tendon could be retracted proximally to allow for fracture site visualization. The soleus was then detached from dorsal fibula with carefulness. Thereafter, the exposed capsule was incised to allow for the visualization of the entire lateral aspects of tibial plateau, including the posterolateral corner.

Temporary fix the fracture fragments with multiple K-wires. After elevation, the defect was filled with artificial bone graft. Then, screw formation of the posterolateral anatomic locking plate was attempted. Three "raft" proximal row locking screws were inserted parallel with the plateau joint surface. The reduction quality and location of internal fixation were further identified by fluoroscopic guidance. The physical examination of the knee stability was routinely conducted to exclude ligament injury.

Mobilization of the knee joint was encouraged immediately after surgery, and discharge was agreed when $90^{\circ}$ was achieved, usually 5-10 days postoperatively. The knee motion range, as well as muscle strengthening was increased after surgical incision healing. Patients were initially allowed no weight bearing for 12 weeks to avoid early loss of reduction. Depending on radiographic signs of healing, partial weight bearing was advised and advanced to full weight bearing as soon as the patients can tolerate.

Clinical evaluation was performed using the TegnerLysholm functional score, Rasmussen functional score [4], and Rasmussen anatomical score [4]. The Rasmussen functional score was used to evaluate knee function primarily with special attention paid to the single plane stability and rotation stability. The Rasmussen functional score is a specific outcome index for postoperative evaluation of tibial plateau fractures. It consists of five categories, including pain (6 points), walking capacity (6 points), extension (6 points), total range of motion (6 points), and stability ( 6 points). The total score is the sum of these five items, and the higher scores indicate the greater function. A score of 30 points is defined as normal, 27-30 as excellent, 2026 as good, 10-19 as fair, and 6-10 as poor. The Rasmussen anatomical score consists of three categories, including depression (6 points), condylar widening (6 points), and angulation (6 points). A score of 18 points is defined as excellent, $12-17$ as good, $6-11$ as fair, and $0-5$ as poor. Medical image data of one patient is shown in Fig. 3.

\section{Results}

The average follow-up was 26 months (12-34 months). The average age of patients was 35 year-old (18-45 years). The general conditions of patients are listed in Table 1 . The mean time from injury to surgery was 6.1 days $(0$ 12 days). At 12 weeks, evidence of radiological fracture union was obtained in all patients (10-14 weeks).

During surgery, time and blood loss were recorded. No case of ligament reparation was found in the study because all patients with knee instability were excluded. Nine patients obtained anatomic articular surface reduction as well as three obtained good reduction.

Tegner-Lysholm function score, Rasmussen functional score, and Rasmussen anatomic score of final follow-up were recorded (Table 2). Radiograph showed intra-articular reduction loss of $2 \mathrm{~mm}$ step-off in one case, which might be caused by weight-bearing too early. No knee instability or pain was found in all cases. The average knee motion arc was $137^{\circ}\left(122^{\circ}-153^{\circ}\right)$.

Incidence of CPN (Common Peroneal Nerve) injury was noted in none case. Lateral knee instability was seen in none case. No flexion contractures or extensor lag was seen. No deep vein thrombosis or graft site morbidity was seen at the follow-up. In addition, no case of incurred nonunion or internal fixation failure was reported. However, 

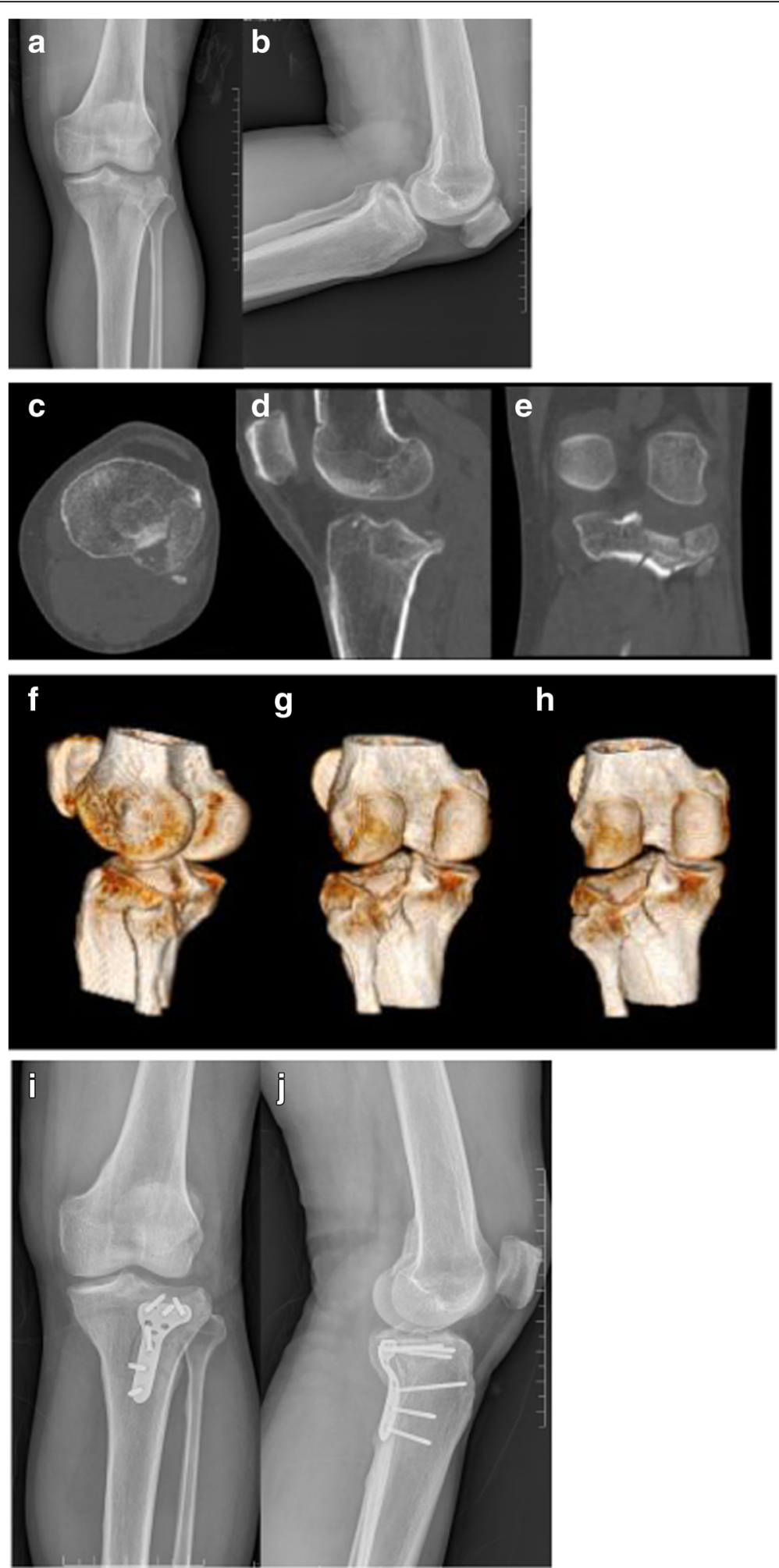

Fig. 3 One male patient with posterolateral tibial plateau fracture, 43y, caused by car-accident. a, b were preoperational anteroposterior and lateral radiographs of one posteroleteral tibial plateau fracture. $\mathbf{c}-\mathbf{h}$ showed more fracture details by 3-D reconstruction of CT scan. $\mathbf{i}, \mathbf{j}$ were anteroposterior and lateral radiographs of the patient acquired after open reduction and internal fixation with the plate 
Table 1 General conditions

\begin{tabular}{lllll}
\hline Case Number & Age $(\mathrm{y})$ & Sex & Cause & Follow-up(mo) \\
\hline 1 & 18 & Male & Veh.Acc. & 12 \\
2 & 43 & Male & Veh.Acc. & 18 \\
3 & 42 & Female & Fall & 32 \\
4 & 38 & Male & Fall & 22 \\
5 & 45 & Male & Fall & 20 \\
6 & 41 & Female & Veh.Acc & 24 \\
7 & 39 & Male & Fall & 34 \\
8 & 35 & Male & Fall & 31 \\
9 & 33 & Male & Veh.Acc & 28 \\
10 & 36 & Female & Fall & 33 \\
11 & 33 & Male & Fall & 27 \\
12 & 31 & Female & Struck & 31 \\
\hline
\end{tabular}

one patient had superficial wound infection that recovered after treatment with antibiotics and local dressing changes.

\section{Discussion}

Patients with untreated posterolateral tibial plateau fracture may suffer from chronic pain, knee instability, and loss of function, especially when climbing the stairs, which even leads to malunion that needs surgery or re-surgery. Thus, many surgeons have agreed that open reduction and internal fixation should be preferred over non-operative treatment $[1,2,4,5]$. Multiple approaches have been described given the complexity of the posterolateral tibial plateau anatomy [2].

Lobenhoffer et al. [9] introduced a posterolateral approach with fibular osteotomy with better visualization. An anterolateral approach with partial or full fibula head removed was developed by $\mathrm{Yu}$ [4], which do great benefit for reduction and fixation in the treatment of posterolateral tibial plateau fractures. However, fibular osteotomies may injury the common peroneal nerve and decrease the stability of the lateral knee joint [11]. Frosch et al. [8] described a posterolateral approach with a lateral arthrotomy for visualizing the joint surface. Posterolateral approach was modified by Tao [7] with an L-shaped incision. Fibular osteotomy was not required in this approach and it protected the soft tissue well, showing encouraging clinical outcomes. The posterolateral approach without fibular osteotomy was chosen in the present study.

Although surgical approaches have been well discussed in previous studies [4, 7-9], operative fixation in treatment of posterolateral tibial plateau fractures has rarely been reported. No specialized plate is used for the treatment due to the irregular shape of the posterolateral tibial plateau. The most widely used system is the distal radius locking plate [1]. The plate usually has to be contoured during surgery to achieve satisfying fitness, which may damage the locking hole and affect the angle stability. Moreover, the plate cannot achieve the "raft" effect due the fact that the proximal locking screws are parallel to the joint surface. This may lead to a higher risk of reduction failure, further comprising outcomes.

Cho et al. [3] reported a novel technique of 2.7- $\mathrm{mm}$ rim plating in tibial plateau fractures to address these problems. Rim plates function as positional plates (named "Hugging plates") rather than buttress plates. The rim plate maintains the posterolateral tibial fracture reduction with the collinear clamp used for a rigid maneuver reduction throughout the fixation procedure. This rim plating technique can be easily applied to isolated posterolateral fracture fragments. However, it is difficult to use in patients with posterolateral tibial comminution and posterolateral corner depression. Moreover,both anterior and posterior stripping to obtain an adequate operative field, together with less antiskid ability, limits its use.

The newly designed anatomic locking plate for the posterolateral tibial plateau, which is oblique " $\mathrm{T}$ " shaped, fits the posterolateral structure of the tibial plateau well

Table 2 Clinical findings

\begin{tabular}{|c|c|c|c|c|c|}
\hline Case Number & Blood loss (ml) & Operation duration (min) & Tegner-Lysholmscore & Rasmussen functional score & Rasmussen anatomical score \\
\hline 1 & 250 & 70 & 97 & 29 & 18 \\
\hline 2 & 100 & 55 & 93 & 28 & 16 \\
\hline 3 & 160 & 55 & 87 & 27 & 14 \\
\hline 4 & 200 & 60 & 89 & 28 & 15 \\
\hline 5 & 230 & 70 & 85 & 25 & 13 \\
\hline 6 & 200 & 65 & 95 & 26 & 16 \\
\hline 7 & 210 & 80 & 90 & 26 & 15 \\
\hline 8 & 240 & 80 & 91 & 28 & 16 \\
\hline 9 & 150 & 75 & 93 & 27 & 16 \\
\hline 10 & 300 & 90 & 88 & 28 & 15 \\
\hline 11 & 180 & 75 & 88 & 27 & 16 \\
\hline 12 & 220 & 70 & 89 & 26 & 15 \\
\hline
\end{tabular}


enough that it reduces plate contouring during surgery, shortens operative duration, decreases blood loss, and potentially decreases plate failure. In our study, our new anatomic locking plate fit anatomically, requiring no additional manipulation. The beveled edges and low profile of the plate can reduce the risk of prominence, which always leads to secondary implant remove surgery. Further, the proximal row locking screws can achieve the "raft" effect, which provides firm strength holding the articular surface. The main limitation distally is the posterior tibial neurovascular bundle. It is difficult to safely achieve exposure beyond approximately $8-10 \mathrm{~cm}$ distal to the lateral joint line owing to vessel trifurcation that transverse the interosseous membrane and muscle mass medial to the fibula. However, the length of plate is appropriate to reach the distal border, ... without damaging the neuromuscular bundle. Thus, distal tibial exposure is not necessarily required. According to the Rasmussen functional/ anatomical score, all patients achieved "good" results. The plate was proven to be safe and effective in a small-sample-size population (12 patients) during a 12- to 34-month follow-up.

The limitations of the study were the relatively small sample size; hence, large-sample studies need to be conducted to prove the efficacy of the technique. In addition, further biomechanical studies are required to evaluate buttress performance, compression forces, and comparative features against traditional buttress line plates via the posterolateral approach.

\section{Conclusions}

In conclusion, the newly designed anatomic locking plate for the posterolateral tibial plateau was proven to be safe and effective in a small-sample-size population (12 patients) during a 12- to 34-month follow-up. The plate fit the irregular posterolateral tibial plateau well, without requiring additional contouring. Therefore, this new plate may be used as a favorable internal fixation choice for treatment of posterolateral plateau fractures.

\section{Abbreviations}

CPN: Common Peroneal Nerve; CT: Computed tomography

\section{Acknowledgments}

We thank LetPub (www.letpub.com) for providing linguistic assistance during the preparation of this manuscript.

\section{Funding}

Research Grant for the Key Disciplines Group Construction Project of Pudong Health Bureau of Shanghai (Grant No. PWZxq2017-11), the Program for Medical Key Department of Shanghai (Grant No. ZK 2015B17), Program for Outstanding Leader of Shanghai (Grant No. 046), Health Science and Technology of Pudong Municipal Commission of Health and Family Planning of Shanghai (Grant No. PW2015A-15).

\section{Availability of data and materials}

The datasets used and analyzed during the study are available from the corresponding author on reasonable request.

\section{Authors' contributions}

BY and DZ designed the plate and provided the data of patients. ZJ, RA, JZ and $\mathrm{XJ}$ analyzed the data. ZJ and RA wrote the paper. All authors read and approved the final manuscript.

\section{Ethics approval and consent to participate}

This study was reviewed and approved by the Institutional Review Board of Shanghai Pudong Hospital (Approval No.2016005). The study was conducted in compliance with the ethical principles originating in or derived from the Declaration of Helsinki and in compliance with Good Clinical Practice Guidelines. All patients provided signed informed consent.

\section{Consent for publication}

Written consent to publish the content of this report along with the accompanying images was obtained from all patients.

\section{Competing interests}

The authors declare that they have no competing interests.

\section{Publisher's Note}

Springer Nature remains neutral with regard to jurisdictional claims in published maps and institutional affiliations.

Received: 21 March 2018 Accepted: 1 August 2018

Published online: 05 September 2018

\section{References}

1. Bhattacharyya T, McCarty LP 3rd, Harris MB, Morrison SM, Wixted JJ, Vrahas MS, et al. The posterior shearing tibial plateau fracture: treatment and results via a posterior approach. J Orthop Trauma. 2005;19:305-10.

2. Wang Y, Luo C, Hu C, Sun H, Zhan Y. An innovative intra-articular osteotomy in the treatment of posterolateral Tibial plateau fracture Malunion. The journal of knee surgery. 2016;

3. Cho JW, Samal P, Jeon YS, Oh CW, Oh JK. Rim plating of posterolateral fracture fragments (PLFs) through a modified anterolateral approach in Tibial plateau fractures. J Orthop Trauma. 2016;30:e362-e68.

4. Yu B, Han K, Zhan C, Zhang C, Ma H, Su J. Fibular head osteotomy: a new approach for the treatment of lateral or posterolateral tibial plateau fractures. Knee. 2010;17:313-8.

5. Waldrop Jl, Macey TI, Trettin JC, Bourgeois WR, Hughston JC. Fractures of the posterolateral tibial plateau. Am J Sports Med. 1988;16:492-8.

6. Hu SJ, Chang SM, Zhang YQ, Ma Z, Du SC, Zhang K. The anterolateral suprafibular-head approach for plating posterolateral tibial plateau fractures: a novel surgical technique. Injury. 2016;47:502-7.

7. Tao J, Hang DH, Wang QG, Gao W, Zhu LB, Wu XF, et al. The posterolateral shearing tibial plateau fracture: treatment and results via a modified posterolateral approach. Knee. 2008;15:473-9.

8. Frosch KH, Balcarek P. Walde T, Sturmer KM. A new posterolateral approach without fibula osteotomy for the treatment of tibial plateau fractures. J Orthop Trauma. 2010:24:515-20.

9. Lobenhoffer P, Gerich T, Bertram T, Lattermann C, Pohlemann T, Tscheme H Particular posteromedial and posterolateral approaches for the treatment of tibial head fractures. Unfallchirurg. 1997;100:957-67.

10. Luo CF, Sun $H$, Zhang B, Zeng BF. Three-column fixation for complex tibial plateau fractures. J Orthop Trauma. 2010;24:683-92.

11. Pires RE, Giordano V, Wajnsztejn A, Oliveira Santana EJ, Pesantez R, Lee MA, et al. Complications and outcomes of the transfibular approach for posterolateral fractures of the tibial plateau. Injury. 2016;47:2320-5. 